\title{
Ground-Based Ultraviolet-Radiation Measurements during Springtime in the Southern Hemisphere
}

F. JAQUE(1), J. O. TOCHO(2), L. F. DA SILVA(3), G. BERTUCCELLI(4), E. CRINO(5), F. CUSSÓ(1), M. A. DE LAURENTIS(6), J. L. HORMAECHEA(8), G. LIFANTE(1), M. G. NICORA (2), H. F. RANEA-SANDOVAL(4), V. VALDERRAMA(9) and G. D. ZOJA(7)

1. Departamento de Física de Materiales, C-IV, Universidad Autónoma de Madrid 28049 Madrid, Spain

2. Departamento de Física, Universidad Nacional de la Plata and CIOp (CONICET-CIC) - La Plata, Argentina

3. Departamento de Física, Universidad Técnica F. Santa María Casilla 110-V, Valparaíso, Chile

4. Instituto de Física Arroyo Seco - Tandil, Argentina

5. Universidad Nacional de San Luis - San Luis, Argentina

6. Observatorio Astronómico Municipal - Mercedes, Argentina

7. Centro Astronómico - Bariloche, Argentina

8. Estación Astronómica - Río Grande, Argentina

9. Departamento de Matemáticas y Física, Universidad de Magallanes, Punta Arenas, Chile

Abstract - We report the first measurements obtained by a network of UV-B detectors established through Argentina and Chile, at locations covering latitudes extending from $53^{\circ} \mathrm{S}$ to $18^{\circ} \mathrm{S}$. Evidence that UV-B increases are detected at these latitudes during the Austral spring 1993 is presented.

Introduction. - The destruction of the stratospheric ozone layer is generally considered one of the most serious environmental problems. It has been recently published that during October 1998 the ozone hole has been the deepest ever recorded [1]. Reduction of the stratospheric ozone layer was unambiguously detected about two decades ago in the Antarctic continent [2,3]. Since then it has been systematically monitored by different means (satellite, balloon soundings and ground station observations [4-6]. One of the most serious content reduction is the increase of biologically effective ultraviolet doses, particularly the so-called ultraviolet $B$ radiation $(280-320 \mathrm{~nm})$ received at the Earth surface, with multiple possible hazards for living species [7]. Despite these consequences, ground stations to check the UV Sun radiation are not very numerous, particularly outside the circumpolar area in the Southern Hemisphere. With the aim of studying whether the ozone depletion over the Antarctic area has extended further, and whether the possibility that UV-radiation increase may begin to affect inhabited regions, a network of UV-B detectors has been established through Argentina and Chile, at locations covering latitudes extending from $53^{\circ} \mathrm{S}$ to $18^{\circ} \mathrm{S}$ (fig.1). Here we report the first measurements obtained by this network providing evidence that UV-B increases are detected at these latitudes during the last Austral spring. 


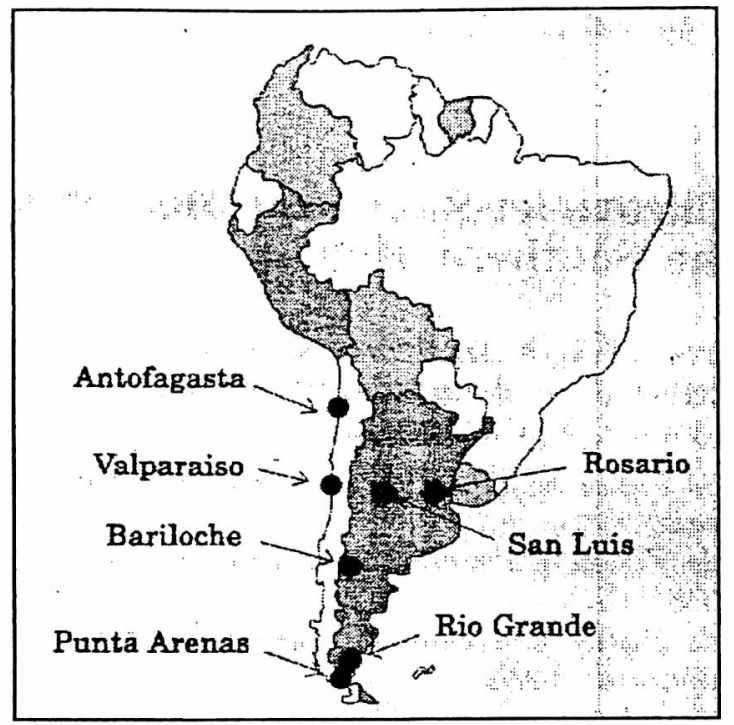

Fig.1 - Location of the ground - based UV-B detectors through Argentina and Chile

Experimental results and discussion. - The devices built for this purpose have been designed to be of low cost and easy to manipulate, in order to implement an extended UV network. The device consists of two EGG detectors (type DF) incorporating blue silicon photodiodes (spectral response $250-900 \mathrm{~nm}$ ) and integrated interferencial filters (centred at $313 \mathrm{~nm}$ and $300 \mathrm{~nm}$, whith $10 \mathrm{~nm}$ bandwidth). Two quartz based telescopic assemblies and an equatorial mounting (ALSTAR) allow Sun tracking for direct solar-radiation measurement. The relative response of the devices has been carefully calibrated at the Centro de Investigaciones Opticas (CIOp), La Plata, Argentine./p>

Due to the substantial difference (a factor of six) in the absorption cross-section at the selected wavelengths ( 313 and $300 \mathrm{~nm}$ ) [8], changes in the irradiance ratio at these wavelengths clearly indicate a decrease in the total ozone amount. Although the irradiance ratio between these two close wavelengths corrects several effects, such as light scattering by clouds [9], all data presented in this work have been taken in clear days, with high horizontal visibility.

Figure 2a) shows the variation of the total ozone during September 1993 (days 245 to 274 of the year), recorded in Punta Arenas (lat. $=52^{\circ} \mathrm{S}$ ), Chile, with a Brewer spectro-photometer, calibrated in Dobson units. It can be observed that a remarkable depletion in the total ozone between the days 250 to 260 was detected, followed by narrow deeps occurring on the days 270 and 276 . The overall ozone reduction registered between the days $248-270$ was about $50 \%$ of the predepletion values.

Figure 2b) shows (circles) the midday irradiance ratio (R) at $313 \mathrm{~nm}$ and $300 \mathrm{~nm}$, taken at the Observatorio Astronómico de Río Grande (lat. $=53^{\circ} \mathrm{S}$ ), Argentina, a locality near Punta Arenas, during the days $246-280$ of 1993 . As can be observed, this ratio shows a pronounced drop in the $\mathrm{R}$ values between the days 250 and 260 , followed by narrow deeps on the days 270 and 276 . The overall decrease registered between the days $246-270$ was about $50 \%$ of the predepletion values. These results are in agreement with the evolution of the total ozone measured in Punta Arenas during the same period of time (fig. 2a). 
continent during the beginning of the 1993 spring.

It is accepted that ozone depletion in the atmosphere is caused by a series of UV induced photochemical and catalytic reactions involving halogens as well as hydrogen and nitrogen compounds ( $\mathrm{HO}^{\mathrm{x}}$ and $\mathrm{NO}^{\mathrm{x}}$ ). The injection of these components, due to the polar-vortex destruction, is generally associated with the recurrent ozone depletion during the Austral spring.

In relation to the UV increase at the Earth surface presented in fig. 2 and 3, the appearance of episodes well localized in time during the days 270 and 276 is apparent. These episodes are of short (few days) duration and appear practically coincident at latitudes as different as in Río Grande (53 $\mathrm{S})$ and Antofagasta $\left(23^{\circ} \mathrm{S}\right)$. Therefore, they seem to be inconsistent with a diffusion process of the "ozone hole" from the Antarctica, and other possibilities must be considered.

It is generally accepted that high-intensity (X) solar flares are capable of depositing large amounts of energy in a short time, involving the production of high concentrations of nitrogen oxide and affecting the ozone concentration at different atmospheric altitudes [10-12], so that this possibility must be considered.

An inspection to the solar-activity reports published by the National Oceanographic and Atmospheric Agency [13,14] reveals the absence of high-intensity (X) solar flares, although two moderate solar flares of magnitude M1.8 on September 27th, and another solar flare (M1.4 intensity) on October 2nd (days 270 and 276) were detected.

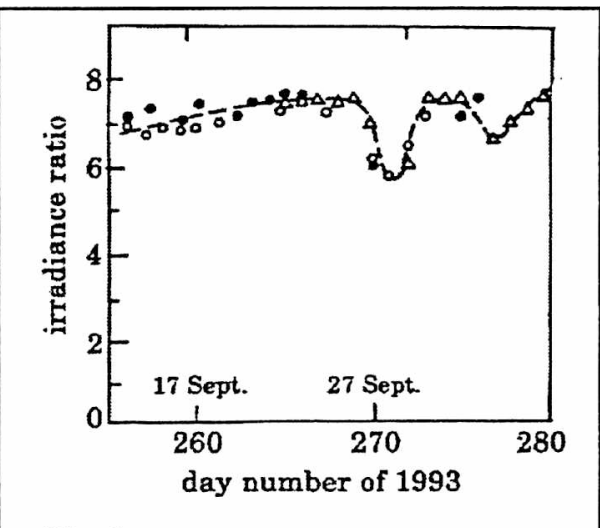

Fig. 3.

Fig. 3 - Evolution of the irradiance ratio at 313 and $300 \mathrm{~nm}$ : Valparaiso (Chile), San Luis (Argentina) and Mercedes (Argentina) during the days 256-280 of 1993.

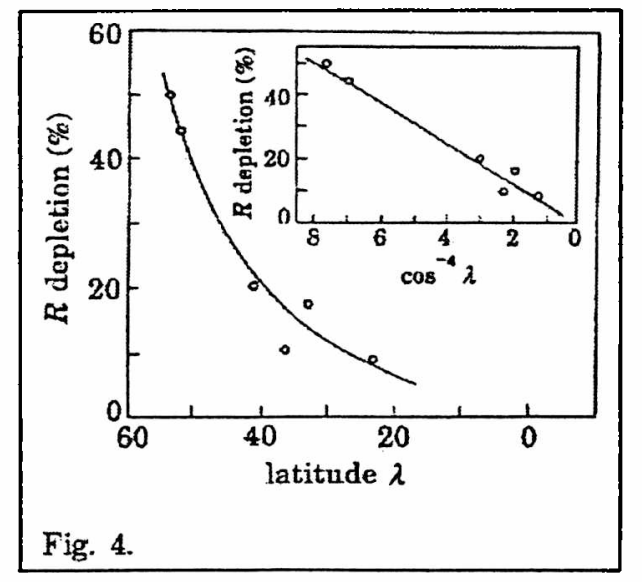

Fig.4 - Dependence of the percentage decrease of the irradiance ratio at $300 \mathrm{~nm}$ and $313 \mathrm{~nm}(\mathrm{R})$ with the latitude 
continent during the beginning of the 1993 spring.

It is accepted that ozone depletion in the atmosphere is caused by a series of UV induced photochemical and catalytic reactions involving halogens as well as hydrogen and nitrogen compounds $\left(\mathrm{HO}^{\mathrm{x}}\right.$ and $\left.\mathrm{NO}^{\mathrm{x}}\right)$. The injection of these components, due to the polar-vortex destruction, is generally associated with the recurrent ozone depletion during the Austral spring.

In relation to the UV increase at the Earth surface presented in fig. 2 and 3, the appearance of episodes well localized in time during the days 270 and 276 is apparent. These episodes are of short (few days) duration and appear practically coincident at latitudes as different as in Río Grande (53 S) and Antofagasta $\left(23^{\circ} \mathrm{S}\right)$. Therefore, they seem to be inconsistent with a diffusion process of the "ozone hole" from the Antarctica, and other possibilities must be considered.

It is generally accepted that high-intensity $(\mathrm{X})$ solar flares are capable of depositing large amounts of energy in a short time, involving the production of high concentrations of nitrogen oxide and affecting the ozone concentration at different atmospheric altitudes [10-12], so that this possibility must be considered.

An inspection to the solar-activity reports published by the National Oceanographic and Atmospheric Agency $[13,14]$ reveals the absence of high-intensity $(X)$ solar flares, although two moderate solar flares of magnitude Ml.8 on September 27th, and another solar flare (M1.4 intensity) on October 2nd (days 270 and 276) were detected.

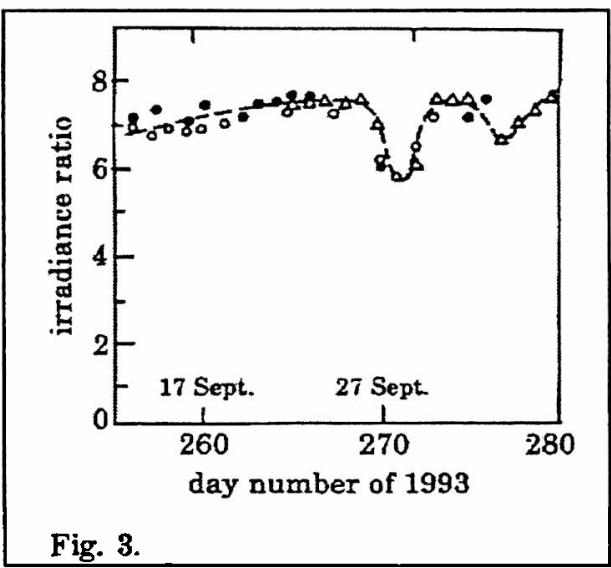

Fig.3 - Evolution of the irradiance ratio at 313 and $300 \mathrm{~nm}$ : Valparaiso (Chile), San Luis (Argentina) and Mercedes (Argentina) during the days 256-280 of 1993.

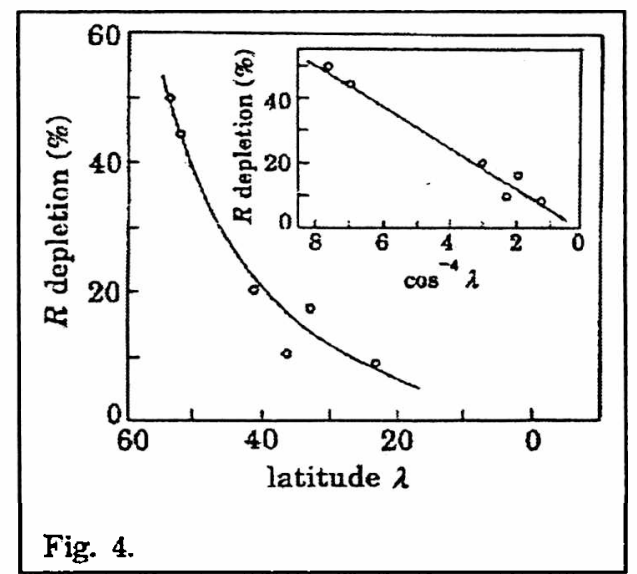

Fig. 4 - Dependence of the percentage decrease of the irradiance ratio at $300 \mathrm{~nm}$ and $313 \mathrm{~nm}$ (R) with the latitude 
(lambda), observed around the day 270 of 1993 at the localities of Punta Arenas, Rio Grande, Bariloche, Tandil, Valparaiso and Antofagasta. The inset illustrates the power dependence. The solid line represents the least-squares fitting of the data.

Some of these less intense events have also been associated to ozone depletion [11], although the connection of this lower intensity (M) events is not as clear as with the high-energy (X) events, which are occasionally associated with a measurable solar-proton event at the Earth surface (Ground-Level Solar Proton Event).

Therefore, although it cannot be unambiguously stated, the coincidence in time suggests a possible connection between solar flares and the UV-radiation increase. We want to remark also that the ozone depletion here reported on days 270 and 276 of 1993 in Punta Arenas amounts to a 50\% of its predepletion value. This is higher than the values previously reported $(23 \%$ [11] and $=5 \%$ [12]) in connection with solar-flare events.

The establishment of a more extensive network, devoted to permanent UV radiation monitoring, covering even higher (equatorward) latitudes than those shown here, would be very convenient to ascertain the origin of the observed UV-radiation increase.

We thank CONICET and CIC-BA (Argentina) and MEC and ICI (Spain) for support. The authors also wish to express their gratitude to Prof. TEITELBAUM and Dr. M. VAZQUEZ for their invaluable suggestions.

\section{References}

1. Walker G., Nature 365 (1993) 683.

2. Chubachi S., in Atmospheric Ozone (Reidel, Dordrecht) 1984, p. 285.

3. Farman J. C., Gardiner B. G. and Shankin J. D., Nature 315 (1985) 207.

4. Gleason J. F., Bhartia P. K., Herman J. R., McPeters R., Newman P., Stolarski R. S., Flynn L., Labow G., Larko D., Seftor C., Wellemeyer C., Kohmyr W.D., Miller A.J. and Planet W., Science, 260 (1993) 523.

5. Hillsenrath E., Attmannspacher W., Bass A., Evans W., Hagemeyer R., Barnes R. A., Kohmyr W., Mauersberger K., Mentall J., Proffitt M., Robbins D., Taylor S., Torres A. and Weinstock E., J. Geophys Res., 91 (1986) 13137.

6. Frederick J. E. and Weatherhead E. C., Photochem. Photobiol., 56 (1992) 123.

7. McKinley A. F. and Diffey B. L., in Human Exposure to Ultraviolet Radiation: Risks and Regulations, edit by W. F. Passchier and B. F. M. Bosnjakovic (Excerpta Medica, Int. Congr. Ser., Amsterdam) 1987, p. 83

8. Molina L. T. and Molina M. J., J. Geophys. Res., 91 (1986) 14501

9. Goody R. M., in Atmospheric Radiation (Oxford University Press, New York, N.Y.) 1964, p. 436.

10. Weeks L. H., Cuikay R. S. and Corbin J. R., J. Atmos. Sci., 29 (1972) 1138.

11. Weath D. F., Krueger A. J. and Crutzen P. J., Science, 197 (1977) 886.

12. Shumilov O. I., Henriksen K., Raspopov O. M. and Kasatkina E. A., Geophys. Res. Lett., 19 (1992) 1647.

13. Solar-Geophysical Data prompt reports, NOAA, Number 590 Part I (1993) 26.

14. Solar-Geophysical Data prompt reports, NOAA, Number 591 Part I (1993) 28. 